Revista Interdisciplinaria de Humanidades, Educación, Ciencia y Tecnología

Año VII. Vol. VII. N¹. Edición Especial. 2021

Hecho el depósito de ley: pp201602FA4721

ISSN-L: 2542-3029; ISSN: 2610-802X

Universidad Nacional Experimental Francisco de Miranda (UNEFM). Santa Ana de Coro. Venezuela

Greys Carolina Herrera-Morales; Mirian Yolanda Jiménez-Gutiérrez

DOI 10.35381/cm.v7i1.468

\title{
Evaluación del Precursor de Carbón Activado Granular del Agua del Río Napo, Comunidad Puerto Colón
}

\author{
Evaluation of the Granular Activated Carbon Precursor from the Napo River \\ Water, Puerto Colón Community
}

\author{
Greys Carolina Herrera-Morales \\ greys.herrera@espoch.edu.ec \\ Escuela Superior Politécnica de Chimborazo, Riobamba \\ Ecuador \\ https://orcid.org/0000-0002-9184-0333 \\ Mirian Yolanda Jiménez-Gutiérrez \\ mirian.jimenez@espoch.edu.ec \\ Escuela Superior Politécnica de Chimborazo, Riobamba \\ Ecuador \\ https://orcid.org/0000-0002-0484-0352
}

Recibido: 30 de marzo de 2020

Aprobado: 15 de junio de 2020 


\begin{abstract}
RESUMEN
El objetivo de la investigación es evaluar el precursor de carbón activado granular para la remoción de contaminantes del agua procedente del rio Napo que se utilizan la Comunidad Puerto Colon, Provincia de Orellana. El estudio evaluó el mejor precursor de carbón activado granular procedente de residuos orgánicos como es de coco, piña y verde. Estos medios filtrantes biodegradables de mayor remoción de contaminantes del agua, es el precursor de carbón activado procedente de la cáscara de cocos nucifera, con $35,3 \%$ en remoción turbiedad, $71,4 \%$ en remoción cadmio y $81,39 \%$ en remoción plomo. La mayor cantidad de precursor es de coco nucifera con $550 \mathrm{~g}$ seguidos de verde con $470 \mathrm{~g}$, y de piña es de $420 \mathrm{~g}$. El análisis de laboratorio del agua del Río Napo, demostró que antes y después de haber filtrado, remueven los contaminantes presentes en el agua; corroborando que la utilización de los filtros biodegradables es menos costosa.
\end{abstract}

Descriptores: Evaluación; carbón; agua. (Palabras del Tesauro UNESCO).

\begin{abstract}
The objective of the research is to evaluate the precursor of granular activated carbon for the removal of pollutants from the water coming from the Napo river used in the Puerto Colon Community, Orellana Province. The study evaluated the best precursor of granular activated carbon from organic waste such as coconut, pineapple and green. These biodegradable filter media with greater removal of contaminants from the water, is the precursor of activated carbon from the shell of cocos nucifera, with $35.3 \%$ in turbidity removal, $71.4 \%$ in cadmium removal and $81.39 \%$ in lead removal. . The largest amount of precursor is coco nucifera with $550 \mathrm{~g}$ followed by green with $470 \mathrm{~g}$, and pineapple is $420 \mathrm{~g}$. The laboratory analysis of the water from the Napo River, showed that before and after filtering, they remove the pollutants present in the water; confirming that the use of biodegradable filters is less expensive.
\end{abstract}

Descriptors: Evaluation; Coal; Water. (Words from the UNESCO Thesaurus). 


\section{INTRODUCCIÓN}

Debido al propio crecimiento de la población humana mundial, esto conllevará a un aumento considerable en la demanda de alimentos y agua potable así como un aumento en la producción industrial. De todos los problemas medioambientales ya existentes y que este crecimiento de la población agravará, el más importante será el de poder disponer de agua potable tanto para la agricultura, ganadería y el consumo humano (Plaza, 2015, p. 1). De esta realidad no escapa la provincia de Orellana, cantón Francisco de Orellana, parroquia García Moreno, que registra problemas relacionados con efectos tóxicos de sustancias en medio natural y no tiene un sistema de agua potable para consumo humano, nada más el $5,4 \%$ recibe el agua de red pública, mientras que $16,5 \%$ lo hacen de pozo, y un elevado $67,4 \%$ de río, vertiente, acequia, agua pluvial o canal (Endavant S.A., 2015, p. 71). Por esta razón se ha evaluado un precursor para el filtro en el que pueda eliminar cualquier impureza y contaminantes presentes en el agua antes de su utilización (Arrocha, 2019, p. 79) que a su vez puede ser útil por la comunidad Puerto Colón.

El precursor de filtro ecológico, es el carbón activo granular procedente de cualquier tipo de carbón granular, madera, lignitos y cáscaras de diversos frutos que tengan propiedades de alta porosidad y dureza para transformar a carbón activo (Bravo Moreira \& Garzón Moreno, 2017, p. 17), como el residuo de cáscara de coco, verde y piña que se obtuvo como precursores de carbón activado granular con la metodología propuesta por Carrillo \& Sánchez (2013); Arrocha (2019); Martínez otros investigadores que lo aplican para recuperar la calidad del agua que ha sido modificada en su composición natural.

Uno de los métodos más desarrollados para la obtención de este precursor es conseguir material procedente de un alto grado de carbón de antracita, donde su proceso se basa en triturar en tamaños pequeños, luego trasladarlo al horno para carbonizar y activar, siendo estos dos pasos simultáneos a temperaturas de 500 grados a 900 grados después de 24 horas aproximadas. La activación dependerá de 
las exigencias de la calidad del carbón activado para que tenga la propiedad de mayor adsorción el carbón activado mayor será la utilización de yodo u otro índice de activación (Endavant S.A., 2015; ONU, UNW-DPAC, s.f.; zhulinacarbon, 2020).

Siendo que para que sea de calidad el carbón activado procedente de materia orgánica será de mayor contenido de porosidad, rico en carbono, dureza, el tamaño del gránulo como por ejemplo un carbón activado de malla o qué es lo mismo de granulometría de $8 \times 30$ que significa un rango de partículas que pasan por la malla número 8 y retenidas en la malla número 30; esto indica que la granulometría o el rango de partícula es de $2.38 \mathrm{~mm}$ hasta $0.595 \mathrm{~mm}$ (Burgos Campuzano y Jaramillo Quiroz, 2015; Carbotecnia, 2020; García López \& Granillo Oporta, 2017; zhulinacarbon, 2020).

Ramírez (2009, pp. 14-15) menciona que el mejor precursor es la concha de coco o cáscara de coco ya que en sus investigaciones el radio medio de poro y radio poro dominante y uniforme con un alto porcentaje de porosidad, excelente para la filtración de aguas contaminadas a más que posee un material rico en carbono con mayor granulación y dureza. El método que utiliza para la activación térmica es en atmósfera saturada de vapor de agua o con gases de combustible.

Entre los objetivos de esta investigación, a partir de la obtención de los precursores de carbón activado es caracterizar el agua del Río Napo, que es la fuente de uso doméstico de esta comunidad. Es así que para el tratamiento del agua procedente de este río se determina análisis de laboratorio antes y después del agua que pasen por estos filtros y a su vez, se concreta la eficiencia de los precursores de carbón activado granular con un análisis comparativo de los resultados de la caracterización físico y química de agua tratada y con la normativa vigente de la Calidad Ambiental y Descarga de Efluentes al Recurso Agua, que detalla en la Tabla 1: Criterios de calidad de efluentes de agua para consumo humano y doméstico (Refórmese el Texto Unificado de Legislación Secundaria, 2015, p. 97). Siendo que, el alcance de esta investigación es mejorar en lo posible la calidad de agua que usan los moradores de la comunidad de Puerto Colón con la utilización de los recursos que estén a su mano. 
Dando una iniciativa para otras investigaciones, siendo un aporte para mejorar la calidad del agua ya sea para consumo humano, utilizada en diversas funciones de las personas que habitan en la región de Orellana o mejorar el hábitat de la fauna y flora acuática del Río Napo.

Ahora bien, dada la importancia de los precursores de carbón activado para el tratamiento del agua, surge esta investigación que tiene como objetivo evaluar el precursor de carbón activado granular para la remoción de contaminantes del agua procedente del rio Napo que se utilizan la Comunidad Puerto Colon, Provincia de Orellana.

\section{MATERIALES Y METODOS}

Con base en el texto de Palella y Martins (2010), los mismos hacen referencia a que "diferentes autores han conceptualizado el método como la manera, el camino que se sigue para lograr un fin. En la investigación, el método implica la elaboración de un plan y la selección de las técnicas más idóneas para su desarrollo" (p.79).

\section{Diseño de la investigación}

El diseño de investigación fue experimental, en el cual el investigador manipula una variable experimentalmente no controlada, bajo condiciones estrictamente controladas. Su objetivo es describir de qué modo y por qué causa se produce o puede producirse un fenómeno (Palella y Martins, p.86). Por su parte, Arias (2006), con relación a este tipo de investigación sostiene "es un proceso que consiste en someter a un objeto o grupo de individuos a determinadas condiciones, estímulos o tratamiento (variable independiente), para observar los efectos o reacciones que se producen (variable dependiente)"(p.83). 


\section{Ubicación de la Investigación}

La investigación se realizó en el cantón Francisco de Orellana situado a una distancia aproximadamente de $300 \mathrm{hm}$ de Quito, capital de Ecuador. Es conocida popularmente como El Coca, con una superficie de 7.047 y con 704.755 de habitantes. Este cantón limita al Norte con el cantón La Joya de los Sachas y la Provincia de Sucumbíos, al Sur con las Provincias Pastaza y Napo, al Este con el cantón Aguarico y al Oeste con el cantón Loreto y la Provincia de Napo (Alcaldía de Francisco de Orellana, 2020). Las coordenadas del área de influencia de la investigación son: LONG: W $77^{\circ}$ 443,776” y LAT: S 0`33'39,354”.

\section{Lógica de la investigación o métodos}

Planteamiento de hipótesis

Hipótesis 1: El precursor de carbón activado granular proveniente de la cáscara de coco es o no, el mejor precursor de carbón activo granular en la filtración del agua de Río Napo.

Hipótesis 2: Si o no se ha removido los contaminantes de agua doméstica mediante filtros ecológicos.

\section{Guía metodológica o técnica de la investigación.}

Número de tratamientos: 3, Número de repeticiones: 4 (Arrocha et al., 2019).

Total, de filtros: 12 filtros $=12$ unidades experimentales

Activación de carbón activado granular: física a temperatura: $500{ }^{\circ} \mathrm{C}$, tiempo aproximado: 3 horas, tamaño granular: $4 \times 10^{6} \mathrm{~nm}$ o $4 \mathrm{~mm}$ (Bravo Moreira \& Garzón Moreno, 2017)

Cantidad de carbón activado granular por filtro: 100 g (Bravo Moreira \& Garzón Moreno, 2017)

Análisis de carbón activado granular: Tamaño granular (Bravo Moreira \& Garzón Moreno, 2017) 
Muestra agua de río Napo: Análisis previo al tratamiento (Arrocha et al., 2019).

\section{Tabla 1.}

Diseño experimental.

\begin{tabular}{|c|c|c|c|c|}
\hline & $\begin{array}{l}\text { Residuo de } \\
\text { coco (cocos } \\
\text { nucifera) }\end{array}$ & $\begin{array}{l}\text { Residuo de piña } \\
\text { (Ananas } \\
\text { comosus) }\end{array}$ & $\begin{array}{l}\text { Residuo de } \\
\text { verde (Musa } \\
\text { paradisiaca) }\end{array}$ & Total muestras \\
\hline $\begin{array}{l}\text { Base } \\
\text { experimental }\end{array}$ & $\begin{array}{l}4 \\
\text { repeticiones: } \\
-1 \text { blanco } \\
-3 \\
\text { repeticiones }\end{array}$ & $\begin{array}{l}4 \text { repeticiones: } \\
-1 \text { blanco } \\
-3 \text { repeticiones }\end{array}$ & $\begin{array}{l}4 \text { repeticiones: } \\
-1 \text { blanco } \\
-3 \text { repeticiones }\end{array}$ & $\begin{array}{l}\text {-12 Unidades } \\
\text { experimentales con } \\
\text { carbón activado } \\
\text { granular }\end{array}$ \\
\hline $\begin{array}{l}\text { Composición } \\
\text { del filtro }\end{array}$ & $\begin{array}{l}\text {-Carbón } \\
\text { activado } \\
\text { granular } \\
\text { (coco) } \\
\text {-Agua del Río } \\
\text { Napo }\end{array}$ & $\begin{array}{l}\text {-Carbón } \\
\text { activado } \\
\text { granular (piña) } \\
\text {-Agua del Río } \\
\text { Napo }\end{array}$ & $\begin{array}{l}\text {-Carbón } \\
\text { activado } \\
\text { granular } \\
\text { (verde) } \\
\text {-Agua del Río } \\
\text { Napo }\end{array}$ & $\begin{array}{l}\text {-misma agua de Río } \\
\text { Napo, } \\
-3 \quad \text { residuos } \\
\text { diferentes }\end{array}$ \\
\hline
\end{tabular}

En la Tabla 1 muestra la base experimental de cada uno de los prototipos de ensayos para asegurar la viabilidad del diseño experimental en los datos obtenidos que revelan cuál de estos precursores de carbón activado granular es un filtro eficiente en la remoción de contaminantes del Río Napo.

\section{RESULTADOS}

A continuación, se muestra los resultados en las tablas de comparación y descripción de cada medida obtenida durante la investigación desarrollada, del carbón activado granular en lo que respecta al tamaño granular, y por último el análisis de turbiedad, $\mathrm{pH}$, cadmio y plomo, antes y después del agua de Río Napo, filtrada en el filtro ecológico. 


\section{Muestras de precursores de carbón activado granular}

La obtención de los precursores de carbón activado granular de cáscara de coco, verde y piña se muestran en la Tabla 2.

\section{Tabla 2.}

Obtención de precursores de carbón activado granular a partir de residuos de coco, verde y piña.

\begin{tabular}{|l|l|l|l|}
\hline & $\begin{array}{l}\text { Residuo } \\
\text { Coco } \\
\text { (Cocos } \\
\text { nucifera) }\end{array}$ & $\begin{array}{l}\text { Residuo Verde } \\
\text { (Musa } \\
\text { paradisiaca) }\end{array}$ & $\begin{array}{l}\text { Residuo Piña } \\
\text { (Ananas } \\
\text { comosus) }\end{array}$ \\
\hline Peso en fresco $(\mathrm{g})$ & 2320 & 2450 & 3710 \\
\hline Peso después de Carbonizado $(\mathrm{g})$ & 580 & 490 & 530 \\
\hline $\begin{array}{l}\text { Peso después de lavado y secado } \\
(\mathrm{g})\end{array}$ & 550 & 470 & 420 \\
\hline $\begin{array}{l}\text { Tamizado 4mm (Tamaño granular) } \\
(\mathrm{g})\end{array}$ & 400 & 400 & 400 \\
\hline $\begin{array}{l}\text { Sobrante del carbón activado } \\
\text { granular (g) }\end{array}$ & 150 & 70 & 20 \\
\hline
\end{tabular}

\section{Muestras de análisis de agua procedente del Río Napo}

\section{Tabla 3.}

Resultados del análisis fisicoquímico de agua natural Río Napo.

\begin{tabular}{|l|l|l|l|l|l|l|}
\hline $\begin{array}{l}\text { Parámetro } \\
\text { Fisicoquímico }\end{array}$ & $\begin{array}{l}\text { Muestra } \\
1\end{array}$ & $\begin{array}{l}\text { Muestra } \\
2\end{array}$ & $\begin{array}{l}\text { Muestras } \\
3\end{array}$ & $\begin{array}{l}\text { Muestra } \\
4\end{array}$ & Promedio & $\begin{array}{l}\text { Límite } \\
\text { permisible }\end{array}$ \\
\hline $\begin{array}{l}\text { Turbiedad } \\
(\text { UNT })\end{array}$ & 124,0 & 152,0 & 162,0 & 98,0 & 134,0 & 100,0 \\
\hline pH & 6,3 & 6,8 & 7,4 & 7,0 & 6,9 & $6-9$ \\
\hline Cadmio (mg/L) & 0,05 & 0,04 & 0,09 & 0,07 & 0,063 & 0,02 \\
\hline Plomo (mg/L) & 0,04 & 0,07 & 0,02 & 0,04 & 0,043 & 0,01 \\
\hline
\end{tabular}




\section{Tabla 4.}

Filtro con precursor de carbón activado granular de residuo de coco (Cocos nucifera).

\begin{tabular}{|l|l|l|l|l|l|l|}
\hline $\begin{array}{l}\text { Parámetros/ } \\
\text { Promedio de las } \\
\text { muestras. }\end{array}$ & $\begin{array}{l}\text { Muestra en } \\
\text { blanco }\end{array}$ & $\begin{array}{l}\text { Muestra } \\
1\end{array}$ & $\begin{array}{l}\text { Muestra } \\
2\end{array}$ & $\begin{array}{l}\text { Muestra } \\
\text { s } \\
3\end{array}$ & $\begin{array}{l}\text { Promedio } \\
\text { por } \\
\text { muestra }\end{array}$ & $\begin{array}{l}\text { Límite } \\
\text { permisible }\end{array}$ \\
\hline $\begin{array}{l}\text { Turbiedad } \\
\text { (NTU) }\end{array}$ & 130,8 & 86,0 & 81,0 & 93,0 & 86,7 & 100,0 \\
\hline $\begin{array}{l}\text { pH (unidades } \\
\text { de } \mathrm{pH})\end{array}$ & 6,6 & 6,7 & 7,1 & 7,0 & 6,9 & $6-9$ \\
\hline Cadmio (mg/L) & 0,050 & 0,019 & 0,012 & 0,022 & 0,018 & 0,02 \\
\hline Plomo (mg/L) & 0,030 & 0,012 & 0,008 & 0,005 & 0,008 & 0,01 \\
\hline
\end{tabular}

Tabla 5.

Filtro con precursor de carbón activado granular de residuo de verde (Musa paradisiaca).

\begin{tabular}{|l|l|l|l|l|l|l|}
\hline $\begin{array}{l}\text { Parámetros/ } \\
\text { Promedio de } \\
\text { las muestras. }\end{array}$ & $\begin{array}{l}\text { Muestra } \\
\text { en } \\
\text { blanco }\end{array}$ & $\begin{array}{l}\text { Muestr } \\
\mathbf{a} \\
\mathbf{1}\end{array}$ & $\begin{array}{l}\text { Muestr } \\
\mathbf{a}\end{array}$ & $\begin{array}{l}\text { Muestras } \\
\mathbf{2}\end{array}$ & $\begin{array}{l}\text { Promedio } \\
\text { por } \\
\text { muestra }\end{array}$ & $\begin{array}{l}\text { Límite } \\
\text { permisible }\end{array}$ \\
\hline $\begin{array}{l}\text { Turbiedad } \\
\text { (NTU) }\end{array}$ & 132,7 & 114,0 & 96,0 & 125,0 & 111,6 & 100,0 \\
\hline $\begin{array}{l}\text { pH (unidades } \\
\text { de pH) }\end{array}$ & 6,9 & 6,2 & 7,3 & 7,8 & 7,1 & $6-9$ \\
\hline Cadmio (mg/L) & 0,048 & 0,021 & 0,034 & 0,038 & 0,031 & 0,02 \\
\hline Plomo (mg/L) & 0,028 & 0,015 & 0,008 & 0,017 & 0,013 & 0,01 \\
\hline
\end{tabular}


Tabla 6.

Filtro con precursor de carbón activado granular de residuo de piña (Ananas comosus).

\begin{tabular}{|c|c|c|c|c|c|c|}
\hline $\begin{array}{l}\text { Parámetros/ } \\
\text { Promedio } \\
\text { de las } \\
\text { muestras. }\end{array}$ & $\begin{array}{l}\text { Muestra } \\
\text { en } \\
\text { blanco }\end{array}$ & $\begin{array}{l}\text { Muestra } \\
1\end{array}$ & $\begin{array}{l}\text { Muestra } \\
2\end{array}$ & $\begin{array}{l}\text { Muestras } \\
3\end{array}$ & $\begin{array}{l}\text { Promedio } \\
\text { por } \\
\text { muestra }\end{array}$ & $\begin{array}{l}\text { Límite } \\
\text { permisible }\end{array}$ \\
\hline $\begin{array}{l}\text { Turbiedad } \\
\text { (NTU) }\end{array}$ & 132,7 & 98,7 & 124,0 & 118,0 & 113,6 & 100,0 \\
\hline $\begin{array}{l}\mathrm{pH} \text { (unidades } \\
\text { de } \mathrm{pH} \text { ) }\end{array}$ & 6,9 & 6,4 & 6,4 & 6,9 & 6,6 & $6-9$ \\
\hline $\begin{array}{l}\text { Cadmio } \\
\text { (mg/L) }\end{array}$ & 0,048 & 0,029 & 0,033 & 0,041 & 0,034 & 0,02 \\
\hline Plomo (mg/L) & 0,028 & 0,028 & 0,019 & 0,024 & 0,024 & 0,01 \\
\hline
\end{tabular}

\section{Promedios estadísticos de datos analizados después del medio filtrante.}

Resumen de datos promedio de cada precursor de carbón activado granular en el filtro ecológico versus al agua del Río Napo, que se detalla en la Tabla 7.

\section{Tabla 7.}

Promedio de tratamientos de los precursores de carbón activado granular frente al agua del Río Napo.

\begin{tabular}{|l|l|l|l|l|}
\hline & $\begin{array}{l}\text { Turbiedad } \\
\text { (UTN) }\end{array}$ & $\begin{array}{l}\mathbf{p H} \\
\text { (unidades de } \\
\mathbf{p H}\end{array}$ & $\begin{array}{l}\text { Cadmio } \\
\text { (mg/L) }\end{array}$ & $\begin{array}{l}\text { Plom } \\
\mathbf{o} \\
\text { (mg/L } \\
\text { (mg }\end{array}$ \\
\hline $\begin{array}{l}\text { Tratamiento 1. Residuo Coco } \\
\text { (cocos nucifera). }\end{array}$ & 86,7 & 6,9 & 0,018 & 0,008 \\
\hline $\begin{array}{l}\text { Tratamiento 2. Residuo verde } \\
\text { (Musa paradisiaca). }\end{array}$ & 111,6 & 7,1 & 0,031 & 0,013 \\
\hline $\begin{array}{l}\text { Tratamiento 3. Residuo piña } \\
\text { (Ananas comosus). }\end{array}$ & 113,6 & 6,6 & 0,034 & 0,024 \\
\hline Límite Permisible & 100,0 & $6-9$ & 0,02 & 0,01 \\
\hline
\end{tabular}




\section{Cálculo de la remoción de cada variable físico y químico del agua expuesta en el carbón activado granular.}

\%Remoción $=\frac{\% \text { Remoción }=V P i-V P f}{V P i} X 100$ (1) (Bravo \& Garzón, 2017, p. 23)

(1)

Dónde:

\%Remoción

$$
\begin{aligned}
& P_{i}=\text { Parámetro inicial } . \\
& P_{f}=\text { Parámetro final } .
\end{aligned}
$$

\section{\%REMOCIÓN TURBIEDAD}

$$
\begin{gathered}
\% \text { Remoción de coco }=\frac{134-86,7}{134} \times 100=35,3 \\
\% \text { Remoción de verde }=\frac{134-111,6}{134} \times 100=16,71 \\
\% \text { Remoción de piñ } a=\frac{134-113,6}{134} \times 100=15,22
\end{gathered}
$$

\section{\%REMOCIÓN CADMIO}

$$
\begin{gathered}
\% \text { Remoción de coco }=\frac{0,063-0,018}{0,063} \times 100=71,4 \\
\% \text { Remoción de verde }=\frac{0,063-0,031}{0,063} \times 100=50,79 \\
\% \text { Remoción de piña }=\frac{0,063-0,034}{0,063} \times 100=46,03
\end{gathered}
$$

\section{\%REMOCIÓN PLOMO}

$\%$ Remoción de coco $=\frac{0,043-0,008}{0,043} \times 100=81,39$ 


$$
\begin{gathered}
\% \text { Remoción de verde }=\frac{0,043-0,013}{0,043} \times 100=69,76 \\
\% \text { Remoción de piñ } a=\frac{0,043-0,024}{0,043} \times 100=44,19
\end{gathered}
$$

Resumen de los porcentajes de remoción de contaminantes.

\section{Tabla 8.}

Porcentaje de remoción de contaminantes de agua del Río Napo.

\begin{tabular}{|l|l|l|l|l|}
\hline & $\begin{array}{l}\text { Turbiedad } \\
\text { (UTN) }\end{array}$ & $\begin{array}{l}\text { pH } \\
\text { (unidade } \\
\text { s de pH) }\end{array}$ & $\begin{array}{l}\text { Cadmio } \\
\text { (mg/L) }\end{array}$ & $\begin{array}{l}\text { Eficiencia } \\
\text { (\%) }\end{array}$ \\
\hline Tratamiento 1. Residuo Coco & 35,3 & 71,4 & 81,39 & 62,7 \\
\hline Tratamiento 2. Residuo verde & 16,71 & 50,79 & 69,76 & 45,8 \\
\hline Tratamiento 3. Residuo piña & 15,22 & 46,03 & 44,19 & 35,1 \\
\hline Límite Permisible & 100,0 & 0,02 & 0,01 & \\
\hline
\end{tabular}

\section{DISCUSIÓN}

En la evaluación de la obtención del precursor que se muestra en la Tabla 2 se determina que el material con mayor valorización en cantidad de masa del precursor de carbón activado es 580 gramos procedente de la cáscara de coco de 2320 gramos de residuo, en cambio en los precursores de carbón activado de verde y piña es menor de 470 g, y 420 g; por lo cual se necesita de mayor residuo de 2450 gramos y 3710 de piña que es el de mayor cantidad que se necesita para la obtención del carbón activado granular de menor cantidad.

Esto establece que el precursor de carbón activado proveniente de la cáscara de coco ya que es de mayor contenido de material rico en carbono ya que es una fuente algo maderable. Cabe recalcar que todos los precursores cumplen factores de disponibilidad y sin costo de la materia prima, siendo accesible para generarlo.

En cambio, en el análisis del agua procedente del Río Napo se realiza antes y después de pasar por el filtro del cual se obtiene por cada traspaso en los 4 filtros, 48 análisis 
por 4 días de monitoreo aleatorio, como muestra a detalle la Tabla 3. En las Tablas 4, 5, y 6 se describen individualmente la remoción de contaminantes del agua procedente del Río Napo, en cada muestra del medio filtrante que contiene el carbón activado granular ya sea de residuos de coco, verde o piña. En este análisis fisicoquímico del agua del Río Napo por cada toma de muestras en diferentes días se ha determinado un promedio en el cual, el parámetro de turbiedad de 134 UNT, pH de 6.9, cadmio de 0,063 mg/L y plomo de 0,043mg/L, sobrepasan los límites permisibles de la normativa vigente, de la Calidad Ambiental y Descarga de Efluentes al Recurso Agua, que detalla en la Tabla 1: Criterios de calidad de efluentes de agua para consumo humano y doméstico, del Anexo I, Acuerdo Ministerial 097-A.

Se pudo observar en los datos cuantitativos, de cada análisis de laboratorio del agua filtrada procedente del Río Napo, que la disminución de contaminantes de cadmio y plomo como de turbiedad que están dentro de los límites permisibles es el tratamiento de 1 en cual se utilizó como precursor de carbón activado los residuos de cáscara de coco; contrariamente a los otros precursores de carbón activado granular procedentes de piña y verde disminuyen la concentración de contaminantes pero no llegan a estar dentro de los límites permisibles como muestra en la Tabla 7, por ende no enmarcan con la normativa vigente, de la Calidad Ambiental y Descarga de Efluentes al Recurso Agua, que detalla en la Tabla 1: Criterios de calidad de efluentes de agua para consumo humano y doméstico, del Anexo I, Acuerdo Ministerial 097-A.

El mayor porcentaje de remoción de los contaminantes del agua de Río Napo, expuestos en los filtros ecológicos con cada uno de los precursores de carbón activado es el tratamiento 1 de residuos de coco con $62.7 \%$ en la reducción de los contaminantes de cadmio y plomo como de la disminución de turbiedad y $\mathrm{pH}$; seguidamente el tratamiento dos de residuo de verde de $45,8 \%$ y por último $35,1 \%$ en porcentaje de remoción del tratamiento 3 de residuo de piña del cual es muy bajo en la eficiencia para disminuir la concentración y estar dentro de los límites permisibles. 


\section{CONCLUSIONES}

Se caracterizó los parámetros fisicoquímicos del agua de consumo humano y doméstico procedente de la parroquia García Moreno, en la comunidad Puerto Colón, del cual detallan los datos que, el pH, turbiedad, cadmio y plomo, sobrepasan la concentración dentro de los límites permisibles que regula la normativa vigente, de la Calidad Ambiental y Descarga de Efluentes al Recurso Agua, que detalla en la Tabla 1: Criterios de calidad de efluentes de agua para consumo humano y doméstico, del Anexo I, Acuerdo Ministerial 097-A.

Se obtuvieron precursores de carbón activado a partir de los residuos de la cáscara de piña, coco y verde para el tratamiento del agua de consumo humano y doméstico procedente del Río Napo. En el que se aplicó 4 prototipos de diseño experimental de filtros ecológicos.

Se determinó la eficiencia de los precursores de carbón activo granular, siendo el mejor el de residuo como precursor la cáscara de coco tanto en el rendimiento de masa de carbón activo granular obtenido como en la eficiencia de la remoción de contaminantes de agua del Río Napo. Concluyendo así, que el precursor de mayor cantidad y eficiencia en la remoción de contaminantes es todo material rico en carbono, que posee mayor porcentaje de porosidad como de granulometría y dureza, y también el índice de activación es decir con que se aplica al carbón para activarlo después de la carbonización.

Resolviendo así que el interés de esta investigación es dar un producto ecológico y sostenible a la comunidad Puerto Colón y el aporte en la utilización de filtros ecológicos que sean de menor costo y biodegradables para mejorar la calidad del agua y la reutilización de residuos que están activamente generando por la actividad social como económica.

\section{FINANCIAMIENTO}

Monetario. 


\section{AGRADECIMIENTO}

Agradecemos a los colaboradores indirectos de esta investigación, que prestaron las áreas del Laboratorio AQLAB y al Laboratorio de la Faculta de Ciencias, Sede de Orellana de la Escuela Superior Politécnica de Chimborazo.

\section{REFERENCIAS}

Aldana-Zavala, J., y Colina-Ysea, F. Marketing verde en la conformación de una ciudadanía planetaria en el ámbito educativo latinoamericano. Revista San Gregorio, número 31, págs. 150-161. http://dx.doi.org/10.36097/rsan.v0i31.972 (2019).

Arias, F. (2006) El Proyecto de Investigación introducción a la metodología. 5ta. Edición. Editorial Episteme, C.A. Caracas. Venezuela.

Arrocha, F, Guevara, C., González, M., Rivas, F., \& Delgado, R. (2019). Evaluación de filtros de carbón activado basado en cáscaras de frutas (piña, plátano, coco, naranja). Revista de Iniciación Científica, 5, 79-83. https://doi.org/10.33412/revric.v5.0.2390.

Bravo -Moreira, K. I., \& Garzón- Moreno, A. R. (2017). Eficiencia del carbón activado procedente del residuo agroindustrial de coco (Cocos nucifera) para remoción de contaminantes en agua. Escuela Superior Politécnica Agropecuaria de Manabí Manuel Félix López https://n9.cl/ro2g

Burgos -Campuzano, G, \& Jaramillo - Quiroz, J. (2015). Aprovechamiento de los residuos de cacao y coco para la obtención de carbón activado, en el cantón Milagro, provincia del Guayas. Universidad de Guayaquil Facultad de Ciencias Químicas.

Carbotecnia. (2020). Granular activated carbón. https://n9.cl/dqpuk

Endavant S.A. (2015). Actualización del Plan de Desarrollo y Ordenamiento Territorial. http://app.sni.gob.ec/sni 
García - López, R., \& Granillo - Oporta, Y. A. (2017). Evaluación de las condiciones operacionales en el proceso de preparación de carbón activo de cáscara de naranja valencia [Citrus Sinensis Linn Osbeck]. Laboratorios de Química UnanManagua, II Semetre 2016. [Facultad De Ciencias E Ingeniería Departamento De Química, Universidad Nacional Autónoma De Nicaragua Unan-Managua Recinto Universitario Rubén Darío].

ONU, UNW-DPAC. (s. f.). Implementación de mejoras para la calidad del agua y la protección de servicios ecosistémicos. Recuperado 1 de enero de 2021, de https://n9.cl/cxufa

Palella, S. Y Martins, F. (2010) Metodología de la investigación cuantitativa. [Quantitative research methodology].3ra edición. Editorial FEDUPEL. Caracas.

Plaza-Recobert, M.(2015). Carbón activado. Evaluación de nuevos precursores y del proceso de activación con dióxido de carbono. Revista Dialnet. España.

Ramírez - Guerra, C. A. (2009). El Carbón activado para el tratamiento del agua. Universidad de Sonora. Departamento de Ingeniería Química y Metalurgia. https://n9.cl/byuxp

zhulinacarbon. (2020). Granulometría o número de malla, Standard Sieve Mesh. Carbotecnia. https://n9.cl/vhec 